\title{
Die ambulante Soziotherapie nach § 37a SGB V ist gescheitert
}

\author{
Outpatient Sociotherapy According to German Code of Social Law V Failed
}

Autoren

Pro: Wulf Rössler, Heiner Melchinger

Kontra: Sibylle Schreckling

\section{Bibliografie}

Dol http://dx.doi.org/

10.1055/s-0032-1304856

Psychiat Prax 2012; 39:

106-108

(c) Georg Thieme Verlag KG

Stuttgart · New York

ISSN 0303-4259

Korrespondenzadressen Prof. Dr. med. Dipl.-Psych. Wulf Rössler

Psychiatrische Universitätsklinik, Klinik für Soziale Psychiatrie Militärstraße 8 / Postfach 1930 8021 Zürich, Schweiz roessler@dgsp.uzh.ch

\section{Dr. Sibylle Schreckling}

für den Berufsverband der Soziotherapeuten Dieselstraße 4

50354 Hürth

info@schreckling.eu

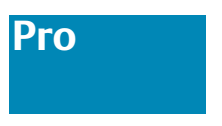

Die Unzulänglichkeiten des gegliederten Systems der sozialen Sicherung in Deutschland schlagen sich insbesondere bei der Behandlung und Betreuung psychisch kranker Menschen nieder. Der Entstehungszusammenhang im Hinblick auf die ambulante Soziotherapie nach §37a SGB V ist hierfür ein klassisches Lehrbeispiel.

Die 90er-Jahre des letzten Jahrhunderts waren vor dem Hintergrund der großen Psychiatrie-Modellprogramme der 70er- und 80er-Jahre zunächst von einer gewissen Erschöpfung im Hinblick auf die Weiterentwicklung der psychiatrischen Versorgung geprägt. Gleichwohl setzte sich langsam die Erkenntnis durch, dass neben dem Aufbau von Sonderinstitutionen wie sozialpsychiatrischen Diensten und psychiatrischen Institutsambulanzen zur Versorgung schwerer psychisch Kranker mit komplexem Versorgungsbedarf auch die medizinische Regelversorgung gestärkt werden müsste.

Ambulante Soziotherapie sollte der Testfall werden. Der Grundgedanke der ambulanten Soziotherapie war, niedergelassenen Nervenärzten/ Psychiatern die Möglichkeit zu geben, unter Einbezug von Soziotherapeuten auf die Bedarfe von Patienten zugeschnittene Komplexleistungen in ihr Behandlungsprogramm zu integrieren.

Der gesetzlichen Verankerung von Soziotherapie ging ein vom Bundesministerium für Gesundheit initiiertes und in Trägerschaft der Spitzenverbände der gesetzlichen Krankenkassen durchgeführtes Modellprojekt „Ambulante Rehabilitation psychisch Kranker“" voraus. Die beteiligten Krankenkassen waren von Beginn an eher widerstrebend, einen solchen Modellversuch durchzuführen. So wehrte sich der Bundesverband der AOK dagegen, dass die Krankenkassen alleine mit der Finanzierung von Soziotherapie belastet werden sollten. Für die damalige Vorstandsvorsitzende der AOK Niedersachen war Soziotherapie der Versuch einer Verlagerung von Leistungen der öffentlichen Hand zulasten der Krankenkassen.

In dem 3-jährigen Modellprojekt konnte der Nachweis geliefert werden, dass Soziotherapie ein praktikables und wirksames Instrument zur Rezidivprophylaxe darstellt. Es konnte auch belegt werden (soweit dies in einem Projekt mit nur 3-jähriger Laufzeit möglich ist), dass den Ausgaben für Soziotherapie ganz erhebliche Einsparungen an stationären Behandlungskosten gegenüberstehen [1].

Soziotherapie sollte im Rahmen des Gesamtpakets der Gesundheitsreform 2000 gesetzlich verankert werden. Für viele überraschend tauchte aber Soziotherapie im Referentenentwurf zur Gesundheitsreform 2000 nicht auf. Durch Intervention auf verschiedenen politischen Ebenen wurde Soziotherapie in letzter Minute doch noch als $§ 37$ a SGB V in den Gesetzentwurf aufgenommen. Es dauerte 2 Jahre bis nach einem quälend langen Diskussionsprozess die Durchführungsrichtlinien zu diesem Gesetz vorlagen. Mit Wirkung zum 1. Januar 2002 war damit Soziotherapie endgültig verordnungsfähig.

Durch die Durchführungsrichtlinien und durch die nachfolgenden „Begutachtungsrichtlinien Ambulante Soziotherapie“ des Medizinischen Dienstes der Krankenversicherung zieht sich wie ein roter Faden das Bemühen, den Umfang der Soziotherapie-Verordnungen möglichst gering $\mathrm{zu}$ halten und Leistungen auszuschließen, die der Leistungspflicht der Sozialhilfe oder anderer Kostenträger zugeordnet werden können.

Eine rigide Einschränkung von Durchführungsmöglichkeiten wurde von den Krankenkassen schon bei der Einführung der „Rehabilitationseinrichtung für psychisch Kranke (RPK)“ praktiziert. Ein nach jahrelangen zähen Verhandlungen zwischen den beteiligten Sozialleistungsträgern gefundener Kompromiss wurde von den Krankenkassen durch plötzliche Vorgabe eine Mindest- 
größe von 50 PRK-Plätzen torpediert, wodurch die als gemeindenahe Einrichtung konzipierte RPK zu einer gemeindefernen Einrichtung werden musste.

Eine Variante der ambulanten Soziotherapie ist im Kleide der integrierten Versorgung wieder auferstanden. Die Krankenkassen sehen in der integrierten Versorgung v. a. die Möglichkeiten zur Kostenreduktion durch Verhinderung (unnötiger) stationärer Aufenthalte, was in der Ausformulierung der Richtlinien für die ambulante Soziotherapie schon als vorrangiges Ziel genannt wurde.

Die Psychiatrie hat sich dem Modell der integrierten Versorgung nur sehr zögerlich angenommen. Alle Beteiligten hatten sich inzwischen einigermaßen komfortabel in der psychiatrischen Versorgung eingerichtet. Das Modell der integrierten Versorgung, so wie es gegenwärtig in Niedersachsen erprobt wird, ermöglicht ein Komplexleistungsprogramm für schwerer psychisch Kranke durch den koordinierten Einsatz niedergelassener Psychiater und ambulanter psychiatrischer Pflegedienste. Aber auch in Niedersachsen gibt es bisher nur relativ wenige Orte, wo ambulante psychiatrische Krankenpflege angeboten wird. In den Flächenländern Sachsen, Thüringen, Bayern, Schleswig-Holstein und Mecklenburg-Vorpommern gibt es jeweils nur einen einzigen Dienst, der die Zulassung zu ambulanter psychiatrischer Pflege erhalten hat.

Seit 10 Jahren ist Soziotherapie verordnungsfähig. Bis heute ist es in den meisten Bundesländern nicht gelungen, eine flächendeckende Versorgung mit Soziotherapie auch nur in Ansätzen zu erreichen [2]. Den Großstädten, wo Soziotherapie am ehesten zum Einsatz kommt, stehen große Flächenregionen gegenüber, in denen Soziotherapie noch nie oder nur in wenigen Fällen verordnet wurde.

Zur Verfügbarkeit von Soziotherapie ein paar Daten, jeweils bezogen auf 100000 Einwohner: Die Anzahl zugelassener Soziotherapeuten variiert zwischen den Bundesländern um den Faktor 40 (von 0,05-2,0). Tatsächlich bewilligte Soziotherapiefälle variieren zwischen 0,5 und 30 Fällen, wobei nur in 3 Bundesländern mehr als 10 Fälle/100000 EW erreicht werden [3]. In BadenWürttemberg nahmen die Anträge auf Gewährung von Soziotherapie von 2006-2010 um 27\% ab, die Zahl der Bewilligungen um $19 \%$ [4]. Auch aus anderen Bundesländern wird die Abnahme von Soziotherapiefällen berichtet.

Im Zuge des Gesetzgebungsverfahrens zur Soziotherapie wurde die maximale Mehrbelastung der GKV durch Gewährung von Soziotherapie auf 125 Mio. im ersten und auf 250 Mio. im zweiten Jahr nach Einführung geschätzt. Im Jahr 2008 betrugen die Ausgaben der GKV für Soziotherapie rd. 3,4 Mio. Euro und damit $1,4 \%$ (!) der prognostizierten 250 Mio. €.

Das Scheitern der Soziotherapie war durch 2 Bedingungen vorprogrammiert: durch unrealistisch hohe Ansprüche an die Qualifikations- und Erfahrungsprofile der zuzulassenden Leistungserbringer und durch prohibitiv niedrige Vergütungssätze. Die Stundenvergütungen der Soziotherapeuten variieren zwischen $28 €$ in Thüringen und 44,90 € in Bremen. In vielen Diensten war die soziotherapeutische Leistungserbringung bisher nur durch Querfinanzierung aus anderen Leistungsbereichen möglich, was kein tragfähiges Finanzierungsmodell sein kann. Die verordnungsberechtigten Nervenärzte/Psychiater beklagen einen zu hohen administrativen Aufwand für die Antragstellung und eine nicht annähernd kostendeckende Vergütung für die von den Durchführungsrichtlinien geforderten Abstimmungen mit den soziotherapeutischen Leistungserbringern.

Was bleibt, wenn wir uns aus den Niederungen der Interessenpolitik begeben? Seit 30 Jahren wird versucht, ambulante Komplex- leistungsprogramme für schwerer psychisch Kranke in die Regelversorgung einzuführen. Die meisten Ansätze sind gescheitert, angefangen von den Versuchen im Modellprogramm Psychiatrie der Bundesregierung in den 80er-Jahren, Sozialarbeiter in die Praxen niedergelassener Psychiater zu integrieren, was schon daran gescheitert ist, dass niedergelassene Psychiater dieses unternehmerische Risiko nicht tragen konnten oder wollten. Die ambulante Soziotherapie ist gescheitert, weil die Krankenkassen ihr Misstrauen gegenüber diesem Komplexleistungsprogramm nicht überwinden konnten oder wollten. Die ambulante Soziotherapie ist zu einem Nischenprodukt geworden. An wem wird die integrierte Versorgung scheitern, bzw. wessen Interessen steht jetzt dieses Modell entgegen?

Zusammenfassend können wir uns alle fragen, wieso sich die psychiatrische Versorgung immer unter das Primat der Kostenreduktion stellen lässt? Das Credo der Kostenreduktion haben fast alle auf ihre Fahnen geschrieben, während die anderen medizinischen Fächer oft hemmungslos zu expandieren scheinen. Es wäre an der Zeit, dass wir die Qualität der psychiatrischen Versorgung Vorrang gegenüber Kostenaspekten geben.

\section{Kontra}

Vordergründig betrachtet erscheint diese These nicht so abwegig. Gemäß dem Grundsatz des $\S 27$ Abs. 1 SGB V, der beinhaltet, dass den Besonderheiten schwer psychisch Kranker Rechnung zu tragen ist, wurde im Jahr 2000 mit dem §37a SGB V Soziotherapie als Instrument für die ambulante Versorgung und Behandlung schwer psychisch Erkrankter etabliert [5, 6]. Soziotherapie wird bis heute nicht flächendeckend verordnet und eingesetzt. Die Gründe hierfür liegen im Zusammenspiel gebietsübergreifender Behandlungsfelder.

\section{Ziel und Inhalte}

Soziotherapie bedeutet, die Eigenkompetenz der Patienten zu stärken, das Krankheitsbild begreiflich zu machen und Frühwarnzeichen wahrzunehmen. Die medizinischen Inhalte werden gemeinsam mit Nervenarzt, Patient und Soziotherapeut besprochen und mit dessen Hilfe auf eine verständliche Handlungsebene heruntergebrochen. Hierbei wird auf krankheitsbedingtes Verhalten hingewiesen, verbindliche Handlungsempfehlungen und Bewältigungsstrategien aufgezeigt, ggf. mit aufsuchender Unterstützung. Der Unterschied zur Psychotherapie liegt somit im Erkennen des eigenen Verhaltens und der Ermutigung, Änderungen herbeizuführen. Es handelt sich bei Soziotherapie um einen spezifischen Zugang zum Patienten mit dem Ziel, chronisch seelisch Erkrankte zu unterstützen, eine weitgehend eigenständige Lebensführung mit einer psychischen Erkrankung führen zu können (supportive Therapie) [7]. Gemeinsam mit dem behandelnden Nervenarzt/Psychiater, dem Patienten und den Soziotherapeuten wird ein Behandlungsplan erstellt, der auch vom Patienten unterzeichnet wird, d.h. dieser ist von Anfang an als Partner des Behandlungsvertrags eingebunden. Damit wird seine Mitwirkungspflicht gefördert und gewürdigt [8].

Soziotherapie ist zeitlich begrenzt (120 Stunden innerhalb von 3 Jahren, bei Rezidiv Neuverordnung möglich). Die Frequenz der Sitzungen wird durch den Krankheitsverlauf bestimmt. Kriseninterventionen sind jederzeit möglich, d.h. von der Akutpsychiatrie bis hin zur Versorgung schwer chronisch psychisch Kranker ist Soziotherapie ein verlässlicher Partner der Versorgung. Anhand der Verlaufsbesprechungen werden frühzeitig Wege aufgezeigt, einem verbindlichen Behandlungskonzept zu folgen. Dies 
soll die Erkrankten bei der Entfaltung ihrer Fähigkeiten zur Selbstsorge unterstützen. Diese Hilfen erstrecken sich auf das soziale Umfeld sowie auf Hilfen im Berufsleben.

In den S3-Leitlinien „Psychosoziale Therapien bei Menschen mit schweren psychischen Erkrankungen“ wird Bezug genommen zu multiprofessioneller gemeindepsychiatrischer Behandlung. Hier wird unter den Rahmenbedingungen des SGB V auf die o.g. ambulanten Versorgungsinstrumente verwiesen. Soziotherapie wird als Möglichkeit angegeben, eine therapeutische Komplexleistung, wie sie in Kliniken seit Jahrzehnten üblich ist, auch ambulant zu vermitteln und durchzusetzen. Als Ziele werden genannt: Verbesserung der Tagesstrukturierung, Gestaltung sozialer Beziehungen und Selbstsorge. Soziotherapie ist inhaltlich und formal von Maßnahmen der Eingliederungshilfe und anderen Therapieformen wie z. B. Psychotherapie abzugrenzen. Sie ist als integraler Bestandteil einer ärztlich verantworteten Komplexleistung auf ein umschriebenes Ziel ausgerichtet. Sie ist somit wie ambulante psychiatrische Pflege und Ergotherapie ein wichtiger Baustein für eine wohnortnahe komplexe Behandlungsleistung und kann zu anderen Versorgungsformen wie betreutem Wohnen Tagesstättenangeboten und beschützten Arbeitsplätzen hinführen [9].

Allerdings ist Soziotherapie in Deutschland bislang regional sehr unterschiedlich vertreten. Der Berufsverband der Soziotherapeuten mit derzeit 60 Mitgliedern hat unterschiedlichste Erfahrungen gesammelt. Hervorzuheben ist das Berliner Modell. Die Erfahrungen des Vereins für Psychiatrie und seelische Gesundheit in Berlin zeigen, dass die Versorgung im Rahmen von IV-Verträgen einen deutlichen Vorteil für die Patienten bewirkt hat und dass es hierdurch zu einer überzeugenden Zusammenarbeit der Nervenärzte/Psychiater mit dem Komplementärbereich gekommen ist, insbesondere bezüglich Indikationswissen und Beauftragungsprozedere wie auch in der Zusammenarbeit im Einzelfall. Hier besteht eine gute Zusammenarbeit mit der AOK Nordost, wobei auch Schwersterkrankte außerhalb der F20-Diagnosen aufgenommen werden können. Der differenzialtherapeutische und indikationsmäßig ausgeweitete Einsatz von Soziotherapie und häuslicher psychiatrischer Krankenpflege wird auch von anderen Krankenkassen in den integrierten Versorgungsverträgen unterstützt. Die Evaluation der Charité zur multiprofessionellen Versorgung schwer psychisch Erkrankter steht vor dem Abschluss.

Was für die Behandlungspfade psychisch Kranker logisch erscheint, ist im stark gegliederten Gesundheitssystem in der Durchführung erschwert.

Das Gutachten des Gemeinsamen Bundesausschusses von 2008 hat die Gründe für die mangelnde Umsetzung der Soziotherapie offengelegt. Erschwerend ist das langwierige Anerkennungsverfahren der Krankenkassen und die als verbesserungswürdig eingestufte Zusammenarbeit mit dem medizinischen Dienst bezüglich der Inhalte der Soziotherapie. Hinzu kommt die unzureichende Vergütung für Nervenärzte und Soziotherapeuten.

Die Zahlen der Versorgungsforscher (W. Rössler, H. Melchinger) verdeutlichen die Schieflage der Psychiatrie in Deutschland (s. auch $\mathrm{H}$. Melchinger im Neurotransmitter vom 2.11.2011.) Bedingt durch die ungleiche Verteilung von psychiatrischen und psychotherapeutischen Behandlungsformen (ca. 5000 niedergelassene Nervenärzte/Psychiater vs. ca. 18000 ärztliche und psychologische Psychotherapeuten) resultieren in der Realität für den Schwerkranken ca. 30 Minuten Gesprächszeit pro Quartal, für den nichtpsychotischen Patienten bis zu 10 Stunden pro Quartal.

Dennoch darf die schwierige und zeitaufwendige Behandlung schwer und chronisch psychisch Erkrankter finanziell nicht schlechter gestellt sein als Psychotherapie mit nichtpsychotischen Patienten. Diese Diskrepanz verdeutlicht, dass für die Durchführung gesetzlicher Vorgaben auch der finanzielle Rahmen adäquat gestaltet sein muss.

Die Erfolge der Soziotherapie liegen vorwiegend in einer Erleichterung der Arbeit, im verbesserten Informationsfluss, in der Patientenzufriedenheit und positiven Krankheitsverläufen mit weniger Rezidiven. Die Kosten der Behandlung müssen aber für alle Berufsgruppen zumindest gedeckt sein. Die Rahmenbedingungen des Gesetzes von 2000 müssen an die aktuellen Bedingungen angepasst werden.

Die Bedeutung von Soziotherapie als Maßnahme zur Überwindung der Schnittstellen zwischen unterschiedlichen Versorgungs- und Finanzierungsbereichen ist noch nicht hinreichend erkannt und umgesetzt [10].

\section{Fazit}

Die Tatsache, dass Soziotherapie trotz erschwerter Bedingungen seit 2001 kontinuierlich in unterschiedlicher Ausprägung durchgeführt wird, beweist ihren Stellenwert. Insbesondere kann sie auf eine über 10-jährige Erfahrung zurückgreifen.

Zwar gleicht die Soziotherapie zurzeit noch einem Flickenteppich in der Versorgungslandschaft, doch dieser wird sich stetig ausbreiten und zu innovativen Mustern verwoben werden. Hier sind bewährte Modelle vorhanden und vielversprechende neue Ansätze im Kommen.

Soziotherapie ist somit keinesfalls gescheitert, sondern wird sich als Instrument für eine maßgeschneiderte und effiziente Behandlung und Nachsorge weiter etablieren und bewähren.

\section{Literatur}

1 Melchinger $H$, Machleidt W. Ambulante Soziotherapie als Kassenleistung - wann wird sie anwendbar sein? Sozialpsychiatrische Informationen 2004; 2: 35-42

2 Krüger $U$. Theorie und Praxis der ambulanten Soziotherapie. Ergebnisse und Evaluation. Vortrag am 24.02.2010. www.apk-ev.de/Daten bank/downloads/Soziotherapie

3 Köpke M. Zukunft der ambulanten Soziotherapie für psychisch Kranke. Handlungsoptionen der Länder. Vortrag am 24.02.2010. www.apk-ev. de/Datenbank/downloads/Soziotherapie

4 Liga der freien Wohlfahrtspflege e.V. Jahresbericht 2009. Dokumentation der Sozialpsychiatrischen Dienste. SpDi_Dokumentation_2009. pdf

5 Melchinger $H$. Ambulante Soziotherapie Evaluation und analytische Auswertung des Modellprojekts „Ambulante Rehabilitation psychisch Kranker“ der Spitzenverbände der gesetzlichen Krankenkassen. Baden-Baden: Nomos; 1999

6 Frieboes RM. Soziotherapie gemäß § 37a SGB V. der Nervenarzt 2003; 74: $596-600$

7 Schreckling S. Ambulante wohnortnahe Versorgung und Rehabilitation psychisch Kranker in der Vernetzung Band 12 interdisziplinäre Schriften zur Rehabilitation. Ulm: Univ. Verlag; 2004

8 Bergmann F, Roth-Sackenheim C, Schreckling S. Rehabilitation der ambulanten Facharztpraxis. In: Rehabilitation bei psychischen Störungen. München: Urban und Fischer Elsevier GmbH; 2005: 293 - 304

9 Frieboes RM, Schreckling S. Ambulante Soziotherapie (§ 137 a SGB V). In: Rehabilitation bei psychischen Störungen. München: Urban und Fischer Elsevier GmbH; 2005: 3095-3309

10 Godel-Ehrhardt P. „Der Keil in der Drehtür“. Psychosoziale Umschau $2005 ; 3 / 18$ 